\title{
PEDIATRIC RESEARCH
}

\section{Editorial Statement}

Pediatric Research, which serves as the official publication of three closely allied academic societies, the American Pediatric Society, the European Society for Pediatric Research, and the Society for Pediatric Research, is beginning its 27 th year as a journal devoted to the publication of research related to the understanding of normal and pathologic human development. During its brief history, the journal has assumed a leading role among journals reporting clinical and laboratory research pertinent to pediatrics. Its respectable impact factor (the frequency with which articles are cited) and immediacy factor (the fraction of articles cited within their first year of publication) are just two signs of the journal's scientific value. Many changes have taken place with the growth of the journal, and we, as the editors, thought we should take this opportunity to express the policies of the journal and familiarize the readership with some of the recent and proposed changes.

\section{Scope}

The journal wishes to publish the highest quality original articles related to, or reflecting on, the span of human development from the fetus to the adolescent.

Research that enhances our understanding of physiologic and pathologic processes is of prime interest. The range of acceptable research is as broad as that found at international, national, or regional meetings of our sponsoring societies, and no particular subspecialty discipline has priority. Because Pediatric Research serves as a general research journal, articles that apply to more than one discipline are of particular interest, but the central determining factors for acceptance remain the quality and importance of the research. Acceptable studies may use any of a variety of systems including, but not limited to, theoretic models, cell culture, isolated organs, intact animals, and humans. What is most important is that the research address a clear hypothesis or question, or elucidate a mechanism, and that the chosen system and methods be appropriate for the issue under study.

\section{Types of Articles}

Manuscripts describing original scientific investigations may be submitted as regular research articles or articles for rapid publication. The latter are brief articles thought to have unusual scientific importance, and they undergo expedited review.

We also seek to publish concise state-of-the-art reviews and intend to have them as a regular feature of the journal. These articles are selected or solicited from areas in which there are important new developments not widely appreciated by the readership, findings of interest to investigators in multiple disciplines related to pediatrics, or general themes that have not been critically summarized elsewhere. We welcome suggestions for these reviews.

\section{Editorial Board}

The editorial responsibility for the journal resides with two editorial boards, one in Europe and one in North America, which work conjointly to review articles submitted to their respective offices. After a formal search, the editor and proposed editorial board for each office are selected by the trustees of the International Pediatric Research Foundation, which comprises representatives of each of the three sponsoring academic societies. To enhance the efficiency of the editorial process and expedite reviews, it is the policy that the American editorial board and the European editorial board each reside at a single academic institution. The editors and associate editors of each board serve a 5year nonrenewable term.

\section{Review Process}

Authors may submit manuscripts to either editorial office. There is no difference in the editorial policy or review process of the two offices. We generally suggest that articles from Europe go to that office and all other manuscripts be sent to the American office for convenience.

All mansucripts, original investigations and reviews, undergo peer review. Experts familiar with the specific area of investigation are selected by the editors to provide critical review of a manuscript. Every attempt is made to provide insightful, helpful, and unbiased critiques. Accordingly, careful selection of reviewers is of highest priority. This process is facilitated by having a breadth of expertise on the editorial board, suggestions by the authors for expert reviewers, and reference to the data base kept on all reviewers, which provides areas of interest, quality of reviews, and promptness of response. We recognize the need for rapid responses and the editorial office will continue to devote much of its time and effort to accomplish this end. Referees receive regular reminders, reviews are sent by fax when possible, and the efficiency of the review process is constantly monitored by the managing editor. Over the past 10 years, the average response time for receipt of the initial critique has decreased to 6 weeks, which we consider respectable for a journal of this scope, which has reviewers and authors spread throughout the world. We should also stress here that the journal has a short period from acceptance to publication, which is in the 
range of 3 months, and less than that for rapid publications.

Although the response time for review could be shortened by limiting the extent of review, the referees provide the editors with valuable advice about the importance of an article. The reviewers also provide the authors with instructive comments that help reshape their manuscript so that it is more accessible and accurate. These are vital features that we do not wish to compromise.

No single factor determines acceptance of an article; however, qualities that interfere with acceptance are many. In general, original investigations with a stated hypothesis or question, which are well organized and written in English and have not been published in another form (except as an abstract), are acceptable for submission. Case reports, descriptive surveys, or technical reports that have no clear application are rarely suitable unless they provide unusual biologic insights.

After receipt of the comments from the referees, the editors will render a decision, which is forwarded with the formal critiques. Often, the article is not acceptable as submitted, but the editors and referees are willing to reconsider it after revision by the author. This reconsideration is not meant to indicate any assurance that the manuscript will be accepted eventually. The final decision depends on the adequacy of the changes or responses. Accordingly, it is well worth the time and effort for authors to respond in a careful and orderly fashion.

\section{Publication Process}

When an article is accepted, it will be sent to the publisher for formatting, grammatical editing, and printing, a process that can be expedited when the final version of the manuscript is submitted on a diskette. Authors will then receive page or galley proofs and the edited manuscript. The edited version provides an important critique of writing skills from which many of us may benefit, and it is the last chance to check the scientific accuracy of the article before it is published. Review of the manuscript at this stage is the author's responsibility.

\section{Responsibilities of Authors}

It is expected that all authors have read in detail the final version of the submitted manuscript and share full responsibility for what is written. All studies reported that use biologic materials, animals, or human subjects are expected to have been approved by the institutional review committee for animal or human research, to conform to the Declaration of Helsinki: Recommendations for Conduct of Clinical Research (1) or Guiding Principles in the Care and Use of Animals (2), and to acknowledge such approval. The integrity of such a statement is the responsibility of the authors.
Articles are expected to be original reports and the data should not have been submitted or published elsewhere without full disclosure and submission of the other publication (except when data have been reported in abstracts). Any deviations from these policies will be considered potentially fraudulent. Under such circumstances, the editorial board will first ask the corresponding author for a formal explanation. If a satisfactory response is not forthcoming, a more extensive inquiry will be requested from the institution from which the manuscript originates. Decision regarding the manuscript will then be made by the editorial board based on the response to the inquiry. We trust that ethical breeches will remain extraordinarily unusual.

A more subtle issue is the submission of a portion of a project that has been divided into small reports. Commonly, authors choose to submit these short reports that address different aspects of the same study simultaneously to different journals without disclosure. Recently, the editors of seven major pediatric journals published a policy statement regarding this undesirable practice (3). In this statement, they made it clear that they will inform each other whenever such instances occur (3). We recommend that authors read this clear statement and make every effort to abide by the spirit of their commentary.

\section{Our Role}

We recognize that the best way to accomplish our goals of having a journal that serves as a timely forum for the best clinical and laboratory investigations in pediatric research is to attract excellent articles from the readership and provide rapid, insightful, and fair scientific critiques. We think that these aims have been achieved in great measure through the superb stewardship of the previous editors and their editorial boards. We, the current editors, will continue to maintain the high standards of the journal while exploring reasonable approaches to expediting the review process and expanding the breadth and depth of the scientific content. In our quest to achieve excellence of the journal, we genuinely welcome questions, comments, and suggestions. We intend for our editorial offices to be helpful and our editorial boards to be responsive.

George Lister

Pieter J. J. Sauer New Haven Rotterdam

\section{REFERENCES}

1. Bankowski Z, Levine RJ 1993 Ethics and Research on Human Subjects: International Guidelines. Council for International Organizations of Medical Sciences, Geneva, Switzerland, Annex 1

2. Guide for the Care and Use of Laboratory Animals, DHEW Publication No. (NIH) 85-23, Revised 1985, Office of Science and Health Reports, DRR/NIH, Bethesda, MD

3. Bier DM, Fulginiti VA, Garfunkel JM, Lucey JF, Spranger J, Valman B, Zetterström R 1990 Duplicate publication and related problems. Pediatr Res 28:561 